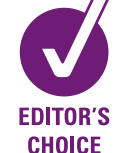

CHOICE

\title{
Microsurgical and endovascular management of pericallosal aneurysms
}

\author{
Ferdinand K Hui, ${ }^{1}$ Albert J Schuette, ${ }^{2}$ Shaye I Moskowitz, ${ }^{1}$ Alejandro M Spiotta, ${ }^{3}$ \\ Michael L Lieber, ${ }^{4}$ Peter A Rasmussen, ${ }^{1}$ Jacques E Dion, ${ }^{5}$ Daniel L Barrow, ${ }^{2}$ \\ C Michael Cawley ${ }^{2,5}$
}

${ }^{1}$ Cerebrovascular Center, Cleveland Clinic, Cleveland, Ohio, USA

${ }^{2}$ Department of Neurosurgery, Emory University, Atlanta, Georgia, USA

${ }^{3}$ Department of Neurosurgery,

Cleveland Clinic, Cleveland

Ohio, USA

${ }^{4}$ Ouantitative Health Sciences, Cleveland Clinic, Cleveland Ohio, USA

${ }^{5}$ Department of Radiology, Emory University, USA

Correspondence to Ferdinand K Hui, Cerebrovascular Center, Cleveland Clinic, 9500 Euclid Avenue, S80, Cleveland, $\mathrm{OH}$ 44195, USA; huif@ccf.org

Received 24 January 2011 Accepted 25 January 2011 Published Online First 1 March 2011

\section{ABSTRACT}

Background Pericallosal, or A2 bifurcation, aneurysms are an infrequently encountered cause of subarachnoid hemorrhage (SAH). While the International Subarachnoid Aneurysm Trial showed improved outcomes for patients with any ruptured anterior circulation aneurysm treated with embolization, there was also a higher recurrence rate for embolized aneurysms. Notably, there were relatively few pericallosal aneurysms.

Objective Specific analysis of pericallosal aneurysms may help guide therapeutic decisions.

Methods Retrospective analysis of patients who presented with proven saccular pericallosal aneurysms was performed at two institutions from 1999 to 2009. Patients were stratified according to presentation Hunt and Hess grades and modified Fisher scores, treatment modality and outcomes as well as development of vasospasm, hydrocephalus and required treatment. Results Eighty-eight patients with pericallosal aneurysms were identified. Sixty-two presented with SAH and 26 in elective fashion, 2 of whom had a prior history of SAH. Fifty-four patients underwent microsurgical repair and 32 endovascular repair. Patients presenting with SAH due to pericallosal aneurysm treated with an endovascular approach were more likely to have a good modified Rankin scale (mRS) (mRS 0-2 vs $3-6)(p=0.028)$, to make a complete recovery $(m R S=0)(p=0.017)$ and were less likely to die $(\mathrm{mRS}=6)(\mathrm{p}=0.026)$. Patients with electively treated pericallosal aneurysms did not have statistically significant differences in outcome between surgical and endovascular cohorts. Differences in secondary endpoints did not reach significance.

Conclusion Patients with ruptured pericallosal aneurysms fare better with endovascular therapy, with better chance of complete recovery. Surgical and endovascular treatments of unruptured pericallosal aneurysms have similar results and outcome.

\section{INTRODUCTION}

Pericallosal aneurysms represent a relatively infrequent cause of subarachnoid hemorrhage (SAH) and are often grouped with other distal anterior cerebral artery (ACA) aneurysms, including callosomarginal artery aneurysms and A3 and A4 branch vessel aneurysms. In the International Subarachnoid Aneurysm Trial (ISAT), there were 95 reported pericallosal aneurysms out of 2143 treated aneurysms, thus comprising $4.4 \%$ of aneurysms. ${ }^{1}$ While the ISAT broadly concluded that there was a benefit of treating ruptured anterior circulation aneurysms in an endovascular fashion, studies comparing endovascular and microsurgical repair in anterior circulation aneurysms occurring at specific locations are lacking. In this study, we reviewed the results of treating pericallosal aneurysms from two institutions spanning 1999-2009.

\section{METHODS}

Institutional review board permission was sought and approved to review all inpatient, outpatient, procedural and radiographic data in these patients.

We retrospectively reviewed 7851 microsurgical and endovascular procedures performed at Emory and at Cleveland Clinic (CCF) from 1999 to 2009 and 2006 to 2009, respectively. The database at CCF did not include sufficient information to warrant inclusion of cases treated prior to 2006. Eighty-eight pericallosal aneurysms were identified out of 4457 cerebral aneurysm cases. Patients were classified according to the presence or absence of $\mathrm{SAH}$, presentation grade with Hunt and Hess $(\mathrm{HH})$ grade and modified Fisher score ( $\mathrm{mFS}$ )

\section{Treatment selection}

All patients diagnosed with a ruptured or unruptured aneurysm underwent a cerebral angiogram to delineate the angioarchitecture of the lesion. In the case of a ruptured aneurysm, the patient and family were offered embolization only if the anatomy was favorable. If so, this was usually the course taken. Note that the exact definition of 'favorable anatomy' has changed with greater operator experience and further advances in endovascular materials. In non-endovascular cases, the patient was taken urgently to the operating room for clip ligation of the aneurysm. Patient preference played a larger role in the treatment of unruptured pericallosal aneurysms. However, once again if the anatomy was favorable for embolization and the patient was agreeable, the patient underwent coiling of the aneurysm.

\section{Surgical technique}

Those lesions felt not amenable to endovascular therapy were referred for surgical repair. In the certain unruptured aneurysms, frameless stereotaxic assistance was occasionally used to help target the craniotomy and dissection. Lumbar drainage was also considered due to the paucity of accessible cisterns for cerebrospinal fluid (CSF) release inherent in the interhemispheric approach. The patient was then placed in a radiolucent head frame to facilitate intraoperative angiography. The patient's torso was kept supine with the head 
Figure 1 Lateral projection of a digital subtraction angiogram of the left internal carotid artery demonstrating a typical pericallosal artery aneurysm before $(A)$ and after (B) clipping.
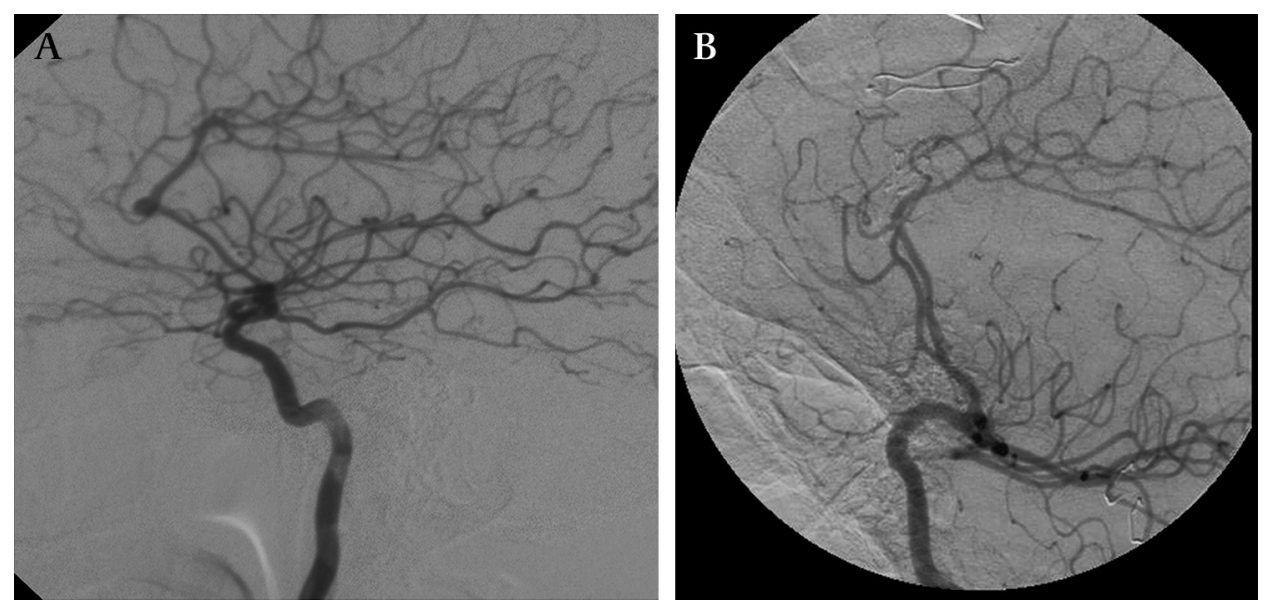

turned either $0^{\circ}$ or $90^{\circ}$, depending on surgeon preference. In either case, the approach was interhemispheric with primary dissection on the right. In the latter case gravity was also employed to allow the frontal lobe to fall away from the falx.

A horseshoe-shaped, or sutar, skin incision crossing the midline was completed midway between the nasion and bregma. After completion of the right frontal craniotomy based just across the midline and straddling the coronal suture, the dura was incised in a U-shaped flap based on the superior sagittal sinus, taking care to avoid any damage to bridging venous complexes. Using the microscope, arachnoidal adhesions between the frontal lobes and falx were separated. Once the fibers of the callosal cistern were identified, both pericallosal and calloso-marginal arteries were followed proximally to the origin of the calloso-marginal artery. Dissection then proceeded along either the anterior or posterior aspect of the pericallosal artery to gain proximal control along the distal A2 or A3 segment. Once this was completed, dissection and clip ligation at the aneurysm neck were performed (figure 1A,B). Temporary clipping was employed to soften the aneurysm or in cases of intraoperative rupture.

\section{Endovascular technique}

Standard microcatheter access to the pericallosal aneurysms treated was achieved while under heparinization per departmental technique. The aneurysms were occluded using coil embolization with materials available at the time, and with balloon remodeling if the neck morphology was challenging (figure $2 \mathrm{~A}, \mathrm{~B})$. Over the course of 10 years, there has been significant evolution of endovascular tools, including the introduction of softer and more trackable microcatheters, microwires with better handling characteristics, smaller and softer coils, as well as balloons for aneurysm neck remodeling. This has in turn increased the proportion of pericallosal aneurysms amenable to endovascular therapy.

\section{Post-procedural care and follow-up}

Aneurysm size and method of treatment were recorded. Angiographic follow-up was obtained at 6, 12 and 24 months after endovascular occlusion, in view of the recurrence rates for endovascular patients. Clinical follow-up was also performed at that time. In the two endovascularly treated patients who were re-treated, one with clip, and the other with muslin wrapping, outcomes were recorded after the re-treatment, with outcomes attributed to the endovascular group.

Patients with SAH were admitted to an intensive care unit until deemed stable for transfer to intermediate care and received a treatment protocol that provided frequent neurological exams, daily transcranial dopplers and repeat imaging for declining neurological status. External ventricular drains (EVDs) were placed in patients with symptomatic hydrocephalus. Nimodipine was administered to all SAH patients. Management of vasospasm included intravenous magnesium infusion, hypertensive, hypervolemic and hemodilutional therapy. Intrathecal nicardipine was also employed in selected patients treated at Emory. Refractory vasospasm was treated with some combination of intra-arterial balloon angioplasty, verapamil or nicardipine. Persistent hydrocephalus was treated with

Figure 2 Lateral projection of a digital subtraction angiogram of the left internal carotid artery demonstrating a typical pericallosal artery aneurysm before $(A)$ and after (B) coiling.
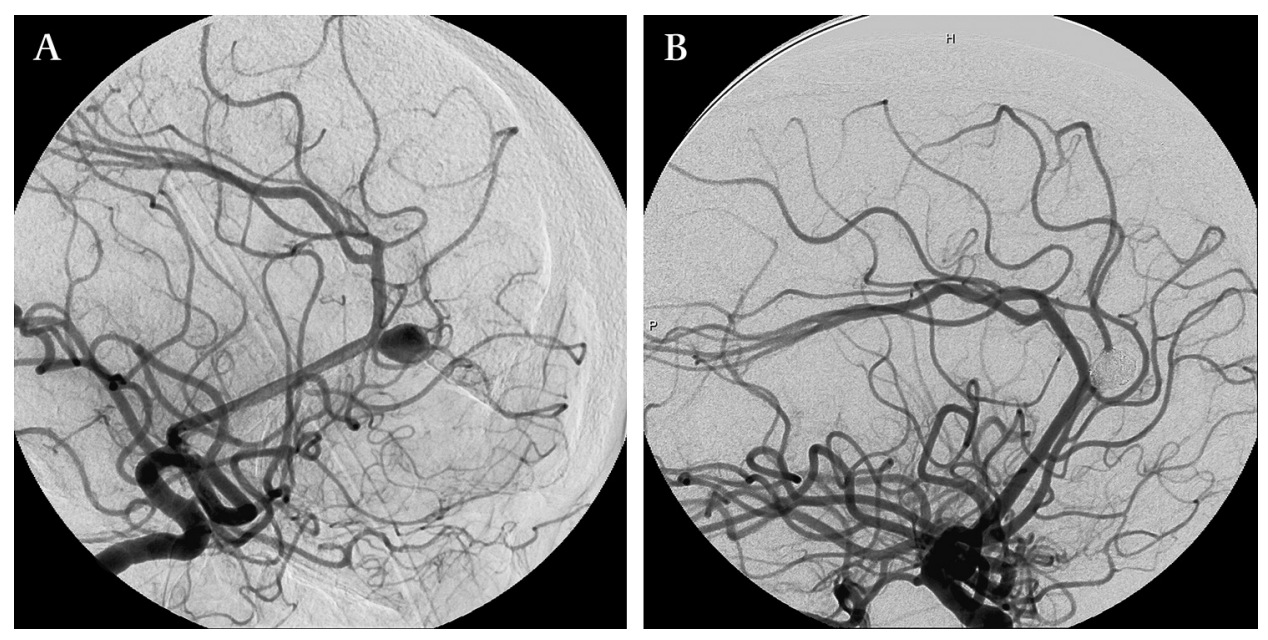
ventriculoperitoneal shunt diversion (VPS), after trials of EVD weaning failed.

Elective aneurysms were treated with routine postoperative management both microsurgically and endovascularly. Endovascular patients were typically discharged the day after the procedure after routine postoperative monitoring. Craniotomy patients were typically discharged on postoperative day 3 or 4 . Outcomes at the latest possible follow-up date were recorded using modified Rankin scale (mRS), typically at 6 months for microsurgical and endovascular patients able to return to clinic. Angiographic follow-up was obtained for endovascular patients up to 2 years post-procedure.

\section{Statistical methods}

The distributions of dichotomous variables and ordinal variables were compared between independent groups using chi-squared tests. ORs are presented where appropriate, along with their corresponding $95 \%$ CIs.

Logistic regression was used to model dichotomized mRS outcomes as a function of therapeutic option (ie, clip versus coil), while simultaneously controlling for the effects of $\mathrm{HH}$ grade, $\mathrm{mFS}$, age, gender and institution.

If a patient had a pericallosal aneurysm that was treated on an elective basis after resolution of a SAH arising from another aneurysm, the patient was considered unruptured for the purpose of the study $(\mathrm{n}=2,2.3 \%)$.

\section{RESULTS}

A total of 88 consecutive pericallosal aneurysms were identified. Relevant demographic characteristics are listed in table 1. A comparison of the demographics between clipped and coiled patients is given in table 2. An analysis of outcomes as measured by $\mathrm{mRS}$ is given in table 3 , and table 4 analyzes clipping versus coiling in ruptured aneurysms looking at the numbers of patients experiencing vasospasm, EVD, shunt placement and death. Figure 3 summarizes outcome distribution for clipping and coiling in ruptured and unruptured aneurysms.

\section{Emory versus CCF}

When comparing the age of both ruptured and unruptured patients, there was a tendency for slightly younger patients at Emory $(n=63$, mean $=50.2)$ versus CCF $(n=25$, mean $=55.2)$ $(p=0.086$; two-sample t-test). When comparing age for electively treated aneurysms and $\mathrm{SAH}$, there was no statistically significant difference for either $(p=0.56$ and $p=0.14$ respectively; two-sample t-test). There was no statistically significant difference when comparing gender of patients at both institutions overall $\left(p=0.37 ; \chi^{2}\right)$ or in either ruptured ( $p=0.44$, Fisher's exact test) or elective ( $p=0.58$; Fisher's exact test) subgroups. Similarly, the mRS distributions for all patients

Table 1 Demographics and description of sample

\begin{tabular}{llc}
\hline & $\mathbf{n}$ & Percentage \\
\hline Total patients & 88 & 100 \\
Male & 16 & 18 \\
Female & 72 & 82 \\
Average age, years & 52 & \\
Clipped & 54 & 61.4 \\
Coiled & 32 & 36.4 \\
Expired prior to treatment & 2 & 2.2 \\
SAH & 62 & 70.4 \\
Non-SAH & 26 & 29.6 \\
\hline
\end{tabular}

Table 2 Clip versus coiling, characteristics

\begin{tabular}{lll}
\hline & Clipped, $\mathbf{n}(\%)$ & Coiled, $\mathbf{n}(\%)$ \\
\hline Totals & 54 & 32 \\
Female & $44(81)$ & $26(81)$ \\
Mean age & 50.2 & 54.4 \\
Aneurysm size & & \\
$\quad<4.0 \mathrm{~mm}$ & $17(32)$ & $8(25)$ \\
$\quad 4.0-8.0 \mathrm{~mm}$ & $31(57)$ & $18(56)$ \\
$\quad>8.0 \mathrm{~mm}$ & $6(11)$ & $6(19)$ \\
Intraoperative rupture & $1 / 54(1.8)^{*}$ & $1 / 32(3.1) \dagger$ \\
Re-treatment & 0 & $2 / 32(6.2)$ \\
Non-SAH & 19 & 7 \\
SAH & 35 & 25 \\
Hunt-Hess grade & & \\
$\quad$ I-II & $16(46)$ & $13(52)$ \\
III & $13(37)$ & $8(32)$ \\
$\quad$ IV-V & $6(17)$ & $4(16)$ \\
Modified Fisher score & & \\
$1-2$ & $6(17)$ & $5(20)$ \\
3 & $10(29)$ & $10(40)$ \\
4 & $19(54)$ & $10(40)$ \\
\hline
\end{tabular}

*The intraprocedural rupture was in a subarachnoid hemorrhage patient (modified Rankin score $(\mathrm{mRS})=2$ )

tThe intraprocedural hemorrhage was in an elective patient $(\mathrm{mRS}=0)$.

between institutions were not significantly statistically different $\left(\mathrm{p}=0.28 ; \chi^{2}\right.$ test, $\mathrm{p}=0.88$ Wilcoxon two sample test), for unruptured patients ( $\mathrm{SAH}-, \mathrm{p}=0.12$; Fisher's exact test, $\mathrm{p}=0.97$; Wilcoxon two sample test), or for ruptured patients (SAH+, $p=0.61$, Fisher's exact test, $p=0.46$; Wilcoxon two sample test). There was no statistically significant difference with regard to treatment modality ratios in all patients $\left(p=0.40 ; \chi^{2}\right)$, for unruptured (SAH-, $\mathrm{p}=0.67$; Fisher's exact test) or ruptured (SAH+, $\mathrm{p}=0.35$; Fisher's exact test).

mFS was not significantly different statistically when comparing institutions (SAH+, $\mathrm{p}=0.33$; Fisher's exact test, $\mathrm{p}=0.17$; Wilcoxon two-sample test). The overall distribution of $\mathrm{HH}$ grades did differ significantly ( $\mathrm{p}=0.034$, Fisher's exact test); however, the Wilcoxon two-sample test showed that the median $\mathrm{HH}$ grade did not differ significantly between the two institutions.

\section{Multivariate analysis}

Multivariable analysis was conducted, showing that when outcomes were dichotomized to $\mathrm{mRS}=0-2$ vs $3-6$, there was a statistically significant difference, with coiling more likely to produce a good outcome in patients with SAH. When this population was dichotomized to $\mathrm{mRS}=0$ vs $1-6$, there was also a statistically significant difference, with embolization more likely to result in a more favorable outcome. When dichotomized to evaluate for likelihood of death $(m R S=6)$, it was also statistically significant. Elective patients did not demonstrate

Table 3 Outcomes

\begin{tabular}{lclllllll}
\hline & \multicolumn{7}{c}{ mRS } \\
\cline { 2 - 8 } & Clip & \multicolumn{7}{c}{ Coil } \\
\hline Elective & 0 & $1-2$ & $3-5$ & 6 & 0 & $1-2$ & $3-5$ & 6 \\
I-II & 6 & 5 & 1 & 0 & 6 & 0 & $1^{*}$ & 0 \\
III & 3 & 2 & 1 & 1 & 9 & 2 & 2 & 0 \\
IV-V & 0 & 1 & 1 & 4 & 3 & 4 & 1 & 0 \\
\hline *One patient had a baseline modified Rankin score (mRS) of 5 from prior subarachnoid
\end{tabular}

*One patient had a baseline modified Rankin score (mRS) of 5 from prior subarachnoid hemorrhage. 
Hemorrhagic stroke

Table 4 Complications and death

\begin{tabular}{|c|c|c|c|c|c|c|c|c|c|c|}
\hline \multirow{2}{*}{ 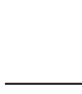 } & \multicolumn{5}{|l|}{ Clip } & \multicolumn{5}{|l|}{ Coil } \\
\hline & Total & VS & EVD & Shunt & Death & Total & VS & EVD & Shunt & Death \\
\hline I-II & 16 & 3 & 4 & 0 & 1 & 13 & 5 & 0 & 0 & 0 \\
\hline III & 13 & 3 & 14 & 2 & 4 & 8 & 0 & 6 & 4 & 0 \\
\hline IV-V & 6 & 1 & 6 & 1 & 4 & 4 & 2 & 2 & 0 & 1 \\
\hline
\end{tabular}

Table organized according to Hunt and Hess grades.

EVD, extravascular drainage; VS, vasospasm.

a statistically significant difference in outcomes. These results are summarized in tables 5 and 6.

Analysis of secondary endpoints was also conducted, comparing EVD, VPS or death, and vasospasm requiring intraarterial therapy and can be found in table 7 . Review of treatment modality demonstrated a trend toward equal utilization of the two treatment approaches in our small series over time (table 8).

\section{DISCUSSION}

Historically, pericallosal aneurysms have more frequently been treated surgically, with a greater number of case series documenting the feasibility of microsurgical treatment ${ }^{2-11}$ when compared with endovascular approaches. ${ }^{12-15}$ However, microsurgical case series have highlighted several significant difficulties in treatment of pericallosal aneurysms from an open approach. ${ }^{1617}$ These difficulties are most apparent when dealing with ruptured lesions, and with aneurysms more proximal relative to the rostrum of the corpus callosum. In such cases, dissection must take place along a very narrow and deep corridor, limited by interhemispheric adhesions and the need to minimize retraction injury to both hemispheres and bridging veins. Furthermore, proximal control is achieved relatively late in the dissection, often after exposing portions of the aneurysm itself. The evolution of modern endovascular techniques now allows the treatment of a much higher percentage of distal ACA aneurysms, and may afford better outcomes for patients.

\section{Limitations}

The non-randomized, retrospective nature of the data intrinsically limits the impact of these findings. SAH cases in which withdrawal of care occurred before diagnostic imaging was

Clip vs Coil, Presentation Grades vs Outcomes, arranged by HH Grade

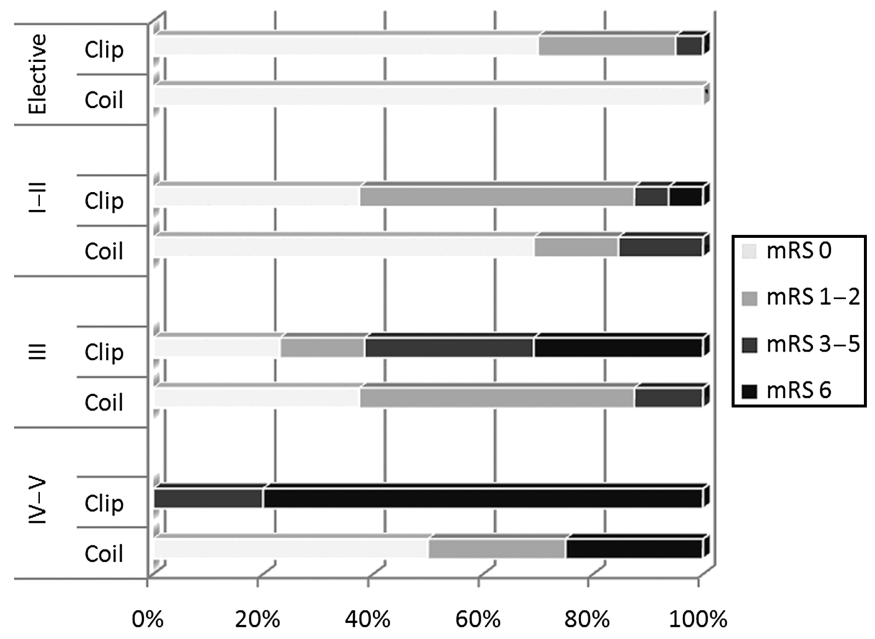

*Note 1 elective coil case was excluded due to a pre-existing $m R S=5$.

Figure 3 Bar graph demonstrating the outcomes from clipped versus coiled aneurysms, stratified by presentation Hunt and Hess grade. $\mathrm{mRS}=$ Modified Rankin Scale
Table 5 Multivariate analysis in electively treated aneurysms

\begin{tabular}{llll}
\hline & Clip, n (\%) & Coil, n (\%) & p-value \\
\hline Elective: clip versus coil, & $18 / 19$ 'good' & $6 / 7(85.7)$ 'good' & $0.44(0.57)^{*}$ \\
mRS 0-2 vs 3-6 & $(94.7)$ & or $6 / 6(100)^{*}$ & \\
Elective: $m R S=0$ & $13 / 19$, & $\begin{array}{l}6 / 7, m R S=0(85.7) \\
\end{array}$ & $0.38(0.11)^{*}$ \\
& $m R S=0(68.4)$ & or $6 / 6(100)^{*}$ & \\
\hline
\end{tabular}

*One patient had a baseline modified Rankin score (mRS) of 5 from prior subarachnoid hemorrhage. The second value is the analysis when excluding that patient.

performed may result in underdiagnosis of pericallosal aneurysms. Additionally, the databases from which these patients were derived do not always clearly indicate pericallosal lesions, which also may result in under-reporting.

\section{Frequency of pericallosal aneurysms}

The number of pericallosal aneurysms collected in our data suggests a rate of $1.8 \%$, which differs from the ISAT data, and may reflect several possibilities. First, the total dataset collected includes many unruptured non-pericallosal aneurysms, which may serve to dilute the number of pericallosals encountered. Second, the rate of rupture of pericallosal aneurysms may be greater relative to other locations, which may explain why the ISAT data have a higher proportion of pericallosal aneurysms. A third possibility may be due to differences in the populations from which these patients are drawn.

\section{Gender of patients with pericallosal aneurysms}

In the ISAT trial, ${ }^{1} 63 \%$ of the $2143 \mathrm{SAH}$ patients were female. In contrast, $82 \%$ of our patients were female. Of the SAH patients in our series, 49/62 (79\%) were female, which appears higher.

\section{Outcomes}

Patients presenting with a SAH due to a pericallosal aneurysm rupture and treated with an endovascular approach were more likely to achieve a good mRS ( $\mathrm{mRS} 0-2$ vs $3-6)(p=0.028)$, to make a complete recovery $(m R S=0)(p=0.017)$ and were less

Table 6 Logistic regression models in subarachnoid hemorrhage patients, $\mathrm{N}=62$

\begin{tabular}{|c|c|c|}
\hline & p-value & OR $(95 \%$ Cl) \\
\hline \multicolumn{3}{|c|}{$\mathrm{mRS}=3-6$ vs $0-2^{*}$} \\
\hline CCF/Emory & 0.40 & \\
\hline Craniotomy & 0.056 & $4.0(0.96$ to 16.7$)$ \\
\hline $\mathrm{HH}$ & 0.03 & 1.9 (1.1 to 3.4$)$ \\
\hline $\mathrm{mFS}$ & 0.21 & \\
\hline Age & 0.33 & \\
\hline \multicolumn{3}{|c|}{$\mathrm{mRS}=1-6$ vs $0 \dagger$} \\
\hline CCF/Emory & 0.43 & \\
\hline Craniotomy & 0.03 & $4.0(1.1$ to 14.5$)$ \\
\hline $\mathrm{HH}$ & 0.17 & \\
\hline $\mathrm{mFS}$ & 0.08 & \\
\hline Age & 0.72 & \\
\hline \multicolumn{3}{|c|}{$\mathrm{mRS}=6$ vs $1-5 \neq$} \\
\hline CCF/Emory & 0.38 & \\
\hline Craniotomy & 0.028 & $18.9(1.4$ to 259.0$)$ \\
\hline $\mathrm{HH}$ & 0.014 & 3.5 (1.3 to 9.5$)$ \\
\hline $\mathrm{mFS}$ & 0.66 & \\
\hline Age & 0.30 & \\
\hline \multicolumn{3}{|c|}{$\begin{array}{l}\text { *The } c \text {-statistic for this model is } 0.81 \text {. Craniotomy (marginally) and higher Hunt-Hess (HH) } \\
\text { grade are associated with a higher risk of modified Rankin Score (mRS) being } \geq 3 \text {. } \\
\text { The c-statistic for this model is } 0.79 \text {. Craniotomy and higher modified Fisher score (mFS) } \\
\text { (marginally) are associated with a higher risk of mRS being }>0 \text {. } \\
\text { FThe c-statistic for this model is } 0.905 \text {. Craniotomy and higher HH grade are both } \\
\text { associated with a higher risk of mRS }=6 \text {. } \\
\text { CCF, Cleveland Clinic. }\end{array}$} \\
\hline
\end{tabular}


Table 7 Extraventricular drain and vasospasm rates

\begin{tabular}{lrlll}
\hline & Crani, $\mathbf{n}(\%)$ & Coil, $\mathbf{n}(\%)$ & $\mathbf{p}$-value & OR (95\% Cl) \\
\hline EVD & $20 / 35(57.1)$ & $8 / 25(32)$ & 0.054 & $0.35(0.12$ to 1.03$)$ \\
VPS or death* & $11 / 35(31.4)$ & $5 / 25(20.0)$ & 0.32 & \\
Vasospasm† $^{2}$ & $6 / 35(17.1)$ & $7 / 24(29.2)$ & 0.27 & \\
\hline
\end{tabular}

*VPS or death, as $\mathrm{mRS}=6$ patients would not receive a shunt.

†Vasospasm requiring intra-arterial therapy.

VPS, ventriculoperitoneal shunt.

likely to die $(m R S=6)(p=0.026)$. The aggregate outcome scores are arranged by percentage and found in figure 2 . All electively coiled pericallosal aneurysms had an mRS of $0(100 \%)$, whereas there was one patient with an mRS of $4(5 \%)$ and five patients (25\%) with mRS of $1-2$ in the clipped group. Of the SAH patients, patients with endovascularly treated aneurysms had $\mathrm{mRS}$ values of $0-284 \%$ of the time, whereas patients with endovascularly treated aneurysms had mRS values of $0-257 \%$ of the time.

Proust et al ${ }^{18}$ summarizes prior case series outcomes regarding microsurgery of aneurysms of the distal ACA, showing 'good outcome' rates ranging between $58.3 \%$ and $94.1 \%$. The patients in most of the series they analyzed had a higher percentage of poor-grade SAH than the series presented here. Lee et al ${ }^{10}$ published a more recent series of 20 distal ACA aneurysms clipped primarily through the standard unilateral interhemispheric approach resulting in 17 with good recovery, 2 deaths and 1 with moderate disability.

With regard to endovascular outcomes, Keston et a ${ }^{15}$ presented their experience in 18 patients, which included seven patients with a mRS of 1 and nine patients with a mRS of 0 ; two patients suffered intraoperative rupture and two patients were not yet followed at the time of publication. Menovsky et al $^{14}$ presented a series of 12 patients, 11 of whom had excellent outcome and one with mild hemiparesis and aphasia.

Patients with electively treated pericallosal aneurysms did not have statistically significant differences in outcome regardless of treatment modality. However, the elective subset had a small sample size and differences may be noted with a larger cohort. Other secondary endpoints such as EVD placement, the development of vasospasm or long-term CSF diversion did not reach significance. Selection bias may influence the distribution of outcomes, as aneurysms that an interventionalist does not deem suitable for coiling may be treated surgically and vice versa.

With regard to result durability, Lahecka et a ${ }^{19}$ have shown no excess long-term mortality in their series of 280 patients with ruptured distal ACA aneurysms, of which $3.6 \%$ were treated

Table 8 Trends in clipping and coiling in two institutions

\begin{tabular}{lcc}
\hline & Clip & Coil \\
\hline 1999 & 2 & 0 \\
2000 & 8 & 1 \\
2001 & 2 & 0 \\
2002 & 2 & 0 \\
2003 & 3 & 1 \\
2004 & 4 & 3 \\
2005 & 6 & 3 \\
2006 & 4 & 7 \\
2007 & 11 & 4 \\
2008 & 7 & 4 \\
2009 & 5 & 9 \\
\hline
\end{tabular}

endovascularly and $93.6 \%$ microsurgically with $2.8 \%$ dying before treatment. Within their series, there were no reruptures in either group. There were no microsurgical recanalizations and $2 / 32(6.2 \%)$ endovascular cases requiring re-treatment. More data on the long-term outcome and durability of aneurysms treated with coiling are necessary.

\section{CONCLUSION}

Both endovascular and microsurgical management of ruptured pericallosal aneurysms are relatively safe and effective, providing outcomes superior to that of the natural history for patients with ruptured aneurysms. Endovascular therapy appears to result in better overall outcomes in the setting of a ruptured aneurysm, similar to that of the ISAT. Eighty-four percent of endovascularly treated patients had a favorable mRS of $0-2$, whereas $57 \%$ of patients treated microsurgically had mRS values of $0-2$. There is no significant difference in the outcomes of electively treated pericallosal aneurysms, although the numbers in this series remain small.

\section{Competing interests None.}

Ethics approval The database was prospectively collected and approved by the IRB office of Cleveland Clinic and Emory University.

Provenance and peer review Not commissioned; externally peer reviewed.

\section{REFERENCES}

1. Molyneux A, Kerr R, Stratton I, et al; International Subarachnoid Aneurysm Trial (ISAT) Collaborative Group. International Subarachnoid Aneurysm Trial (ISAT) of neurosurgical clipping versus endovascular coiling in 2143 patients with ruptured intracranial aneurysms: a randomised trial. Lancet 2002;360:1267-74.

2. Fisher RG, Ciminello V. Pericallosal aneurysms. J Neurosurg 1966;25:512-15.

3. Laitinen L, Snellman A. Aneurysms of the pericallosal artery: a study of 14 cases verified angiographically and treated mainly by direct surgical attack. J Neurosurg 1960:17:447-58

4. Mann KS, Yue CP, Wong G. Aneurysms of the pericallosal-callosomarginal junction. Surg Neurol 1984;21:261-6.

5. Hernesniemi J, Tapaninaho A, Vapalahti M, et al. Saccular aneurysms of the distal anterior cerebral artery and its branches. Neurosurgery 1992:31:994-8; discussion 998-9.

6. Inci S, Erbengi A, Ozgen T. Aneurysms of the distal anterior cerebral artery: report of 14 cases and a review of the literature. Surg Neurol 1998;50:130-9; discussion 139-40.

7. Kwon TH, Chung HS, Lim DJ, et al. Distal anterior cerebral artery aneurysms: clinical features and surgical outcome. J Korean Med Sci 2001:16:204-8.

8. Martines F, Blundo C, Chiappetta F. Surgical treatment of the distal anterior cerebral artery aneurysms. J Neurosurg Sci 1996;40:189-94.

9. Ng PY, Reilly PL, Brophy BP, et al. Distal anterior cerebral artery aneurysms: a clinical series. Br J Neurosurg 1998:12:209-12.

10. Lee JY, Kim MK, Cho BM, et al. Surgical experience of the ruptured distal anterior cerebral artery aneurysms. J Korean Neurosurg Soc 2007:42:281-5.

11. de Sousa AA, Dantas R, Luiz F, et al. Distal anterior cerebral artery aneurysms. Surg Neurol 1999:52:128-36.

12. Pierot $\mathbf{L}$, Boulin A, Castaings $L$, et al. Endovascular treatment of pericallosal artery aneurysms. Neurol Res 1996;18:49-53.

13. Cohen JE, Rajz G, Itshayek E, et al. Endovascular management of traumatic and iatrogenic aneurysms of the pericallosal artery. Report of two cases. J Neurosurg 2005;102:555-7.

14. Menovsky T, van Rooij WJ, Sluzewski M, et al. Coiling of ruptured pericallosal artery aneurysms. Neurosurgery 2002;50:11-15; discussion 14-15.

15. Keston $\mathbf{P}$, White PM, Horribine $\mathrm{L}$, et al. The endovascular management of pericallosal artery aneurysms. J Neuroradiol 2004;31:384-90.

16. Dickey PS, Bloomgarden GM, Arkins TJ, et al. Partial callosal resection for pericallosal aneurysms. Neurosurgery 1992;30:136-7.

17. Traynelis VC, Dunker RO. Interhemispheric approach with callosal resection for distal anterior cerebral artery aneurysms. Technical note. J Neurosurg 1992; 77:481-3.

18. Proust F, Toussaint $P$, Hannequin D, et al. Outcome in 43 patients with distal anterior cerebral artery aneurysms. Stroke 1997;28:2405-9.

19. Lahecka M, Niemela M, Seppanen J, et al. No long-term excess mortality in 280 patients with ruptured distal anterior cerebral artery aneurysms. Neurosurgery 2007:60:235-41: discussion 240-1. 